\title{
Raised CSF phospho-tau concentrations in variant Creutzfeldt-Jakob disease: diagnostic and pathological implications
}

\author{
C A Goodall, M W Head, D Everington, J W Ironside, R S G Knight, A J E Green
}

J Neurol Neurosurg Psychiatry 2006;77:89-91. doi: 10.1136/jnnp.2005.065755

\begin{abstract}
Objective: To investigate whether phosphorylated tau protein (tau-pT181) is increased in variant Creutzfeldt-Jakob disease ( $V C J D)$ and if the tau-pT181/tau protein ratio is useful for distinguishing between patients with and without CJD.

Methods: CSF tau protein and tau-pT181 were measured in 50 patients with sporadic CJD (sCJD), 51 patients with vCJD, $46 \mathrm{sCJD}$ controls, and $37 \mathrm{vCJD}$ controls, using Innotest hTau and Innotest P-Thr181, Innogenetics.

Results: Concentrations of CSF tau protein were increased in sCJD (5120 v $367 \mathrm{pg} / \mathrm{ml}$ in controls, $\mathrm{p}<0.001)$ and vCJD (952 v $106 \mathrm{pg} / \mathrm{ml}, \mathrm{p}<0.001)$; tau-pT181 was also raised in sCJD (61 v $35 \mathrm{pg} / \mathrm{ml}$ in controls, $\mathrm{p}=0.002)$ and vCJD (114 v $33 \mathrm{pg} / \mathrm{ml}, \mathrm{p}<0.001)$. Median concentrations of tau-pT181 were higher in vCJD than in $s$ CJD $(p<0.001)$. The tau$\mathrm{pT} 181 /$ tau protein ratio was lower than in controls in both sCJD $(12 \vee 128(p<0.001))$ and vCJD (119 v 279 $(p<0.001))$. Mean tau-pT181/tau protein ratio was 10 -fold higher in VCJD than in SCJD. Raised CSF tau protein had the highest efficiency for distinguishing SCJD and VCJD from controls.

Conclusions: CSF tau-pT181 concentrations are raised in vCJD and are higher than in SCJD. Measurement of CSF tau$\mathrm{pT} 181 /$ tau protein ratio does not improve the diagnostic efficiency of CSF tau protein alone for either VCJD or sCJD. The higher concentration of CSF tau-pT181 found in VCJD suggests that unexplained pathogenic factors influence the phosphorylation of tau protein in vCJD patients.
\end{abstract}

\footnotetext{
$\mathrm{T}$
} au protein is a microtubule associated protein found in high concentrations in the axons of the central nervous system and is involved in maintaining axonal structure and integrity. ${ }^{1}$ It is normally phosphorylated at six to eight sites, but it can become hyperphosphorylated in various neurodegenerative conditions, such as Alzheimer's disease. ${ }^{2}$ Raised levels of tau protein and phosphorylated tau have been found in sporadic Creutzfeldt-Jakob disease (SCJD) $)^{3-6}$ and a recent study has suggested that the ratio of tau protein to tau phosphorylated at threonine-181 (tau-pTl81) can distinguish SCJD from other forms of dementia. ${ }^{5}$ Raised levels of cerebrospinal fluid (CSF) tau protein have also been found in variant Creutzfeldt-Jakob disease (vCJD) and have been shown to have the greatest diagnostic accuracy of all the CSF proteins investigated for this condition. ${ }^{7}$ In this study we investigated whether measurement of tau-pT181 and the taupTl81/tau protein ratio is better than CSF tau protein alone for the diagnosis of $\mathrm{VCJD}$, and have compared the results with those found in SCJD.

\section{METHODS \\ Patients}

CSF samples from patients referred to the National CJD Surveillance Unit (NCJDSU) were analysed for tau protein and tau-pT181. The methodology of the NCJDSU has been described elsewhere. ${ }^{8}$ All patients or their medical notes, or both, were examined by the NCJDSU. Consecutive patients were selected according to the volume of CSF available for analysis. CSF was analysed from the following patients: 51 patients with neuropathologically proven or clinically probable vCJD, ${ }^{89} 37$ vCJD control patients, 50 patients with neuropathologically proven or clinically probable $\mathrm{sCJD},{ }^{10}$ and 46 SCJD control patients. Thirty two patients had neuropathologically confirmed vCJD (2l male, 11 female; age range 16 to 54 years (mean 30) at notification) and 19 patients had probable vCJD (eight male, 11 female; age range 18 to 50 years (mean 29) at notification). Thirty seven patients had neuropathologically confirmed SCJD (17 male, 20 female; age range 50 to 81 years (mean 63) at notification) and 13 had probable sCJD (six male, seven female; age range 44 to 77 years (mean 66) at notification).

The SCJD and VCJD control groups consisted of patients who were initially suspected of having SCJD and VCJD, respectively, and in whom an alternative diagnosis was made. Of the $37 \mathrm{vCJD}$ control patients ( 16 male, 21 female; age range 14 to 79 years (mean 31 ) at notification), neuropathological examination was carried out on seven cases. The diagnoses in six of these were paraneoplastic syndrome (2), Huntington's chorea (1), multiple sclerosis (1), subacute sclerosing panencephalitis (1), and viral encephalopathy (1). In the remaining case there was no definite neuropathologically confirmed diagnosis on brain biopsy, but no evidence of VCJD. The diagnosis of VCJD was excluded in the remaining 30 vCJD control patients for reasons that included complete or partial recovery (10), or an alternative diagnosis made on clinical grounds, or investigations suggestive of an alternative diagnosis (20). The diagnoses in these 20 cases were psychiatric disorders (7), non-CJD dementia of an unknown cause (5), encephalitis (4), Alzheimer's disease (1), cerebrovascular disease (1), epilepsy (1), and mitochondrial cytopathy (1).

Of the 46 sCJD control patients ( 18 male, 28 female; age range 41 to 84 (mean 68) at notification), 20 had a postmortem neuropathological examination. The diagnoses in 17 of these cases were: Alzheimer's disease (6), Lewy body dementia (2), cerebrovascular disease (2), angiotrophic lymphoma (1), carcinomatosis of leptomeninges (1), cerebral vasculitis (1), ischaemic encephalopathy (1), multifocal demyelination (1), multifocal lymphoma (1), and viral

Abbreviations: tau-pT181, tau protein phosphorylated at threonine181 ; CJD, Creutzfeldt-Jakob disease; sCJD, sporadic CJD; VCJD, variant CJD; NCJDSU, The National CJD Surveillance Unit 

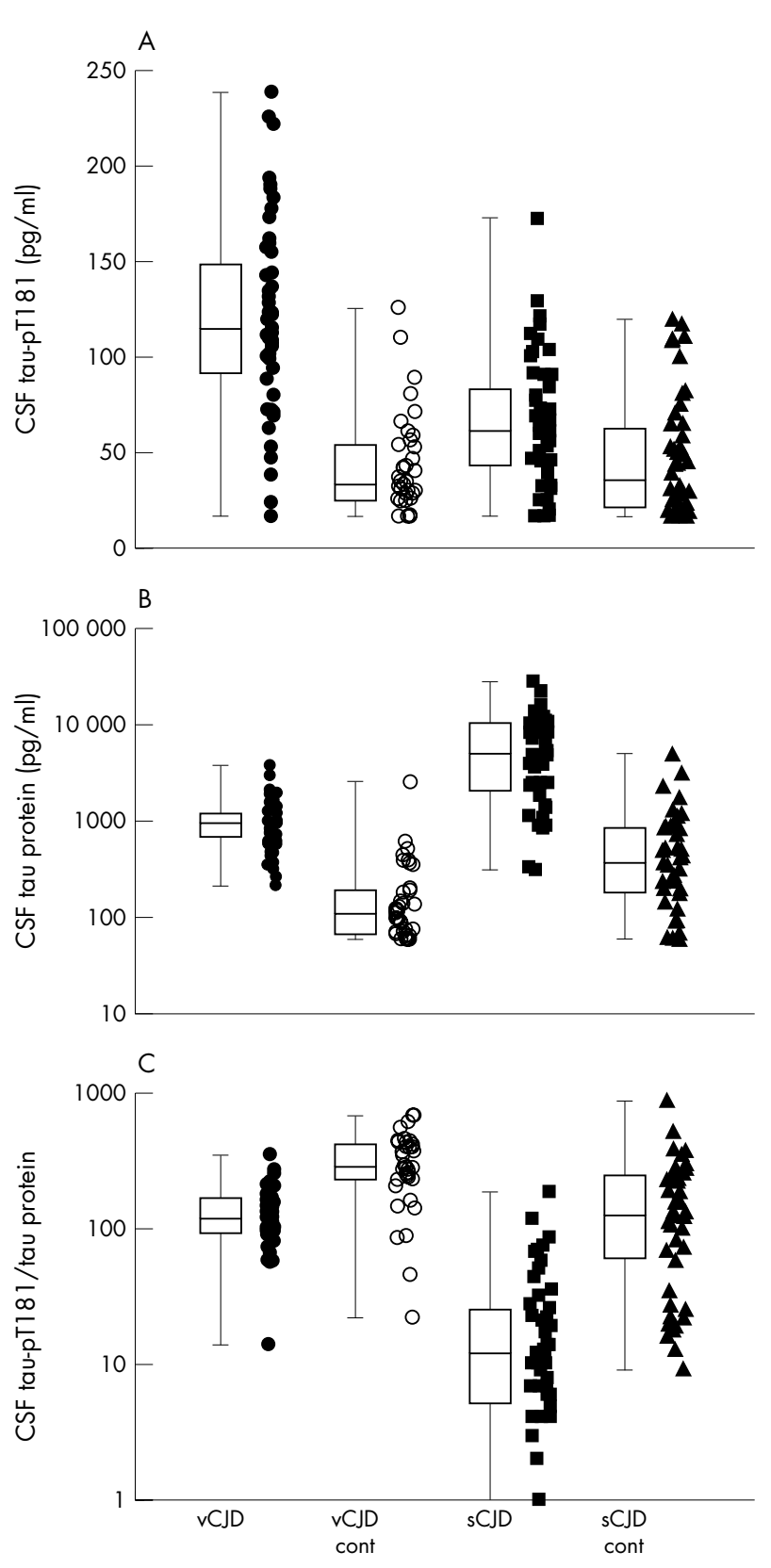

Figure 1 Combined scatter and box plots of CSF tau-pT181 concentrations, CSF tau protein concentrations, and CSF tau-pT181/tau protein ratio $\times 1000$. Variant CJD (VCJD) cases are represented by filled circles, vCJD controls by empty circles, sporadic SCJD cases (sCJD) by filled squares, and sCJD controls by filled triangles. The scatterplots represent the individual values from which the boxes are derived.

encephalitis (1). In the remaining three cases, there was no definite neuropathologically confirmed diagnosis on brain biopsy, but no evidence of SCJD. The diagnosis of SCJD was excluded in the final 26 patients for reasons that include partial recovery (5), or an alternative diagnosis made on clinical grounds, or investigations suggestive of an alternative diagnosis (21). The diagnoses in these 21 cases were: Lewy body dementia (3), non-CJD dementia of an unknown cause (3), frontal lobe dementia (2), Alzheimer's disease (2), Hashimoto's encephalitis (2), paraneoplastic syndrome (2), central pontine myelinolysis (1), cerebrovascular ischaemia (1), granulomatous disease (1), Huntington's disease (1), paraneoplastic encephalitis (1), vascular dementia (1), and vasculitis (1).

\section{CSF analysis}

Aliquots of all CSF samples were taken and stored at $-80^{\circ} \mathrm{C}$ before analysis. CSF tau protein and tau-pT181 were measured using enzyme immunoassays (Innotest hTAU-Ag or Innotest P-Thr181, Innogenetics, Ghent, Belgium). The measurement of both tau protein and tau-pT181 were made according to the manufacturer's recommendations. The taupT181/tau protein ratio was expressed as a ratio of the concentrations multiplied by a factor of 1000 .

\section{Statistics}

Non-parametric tests (Mann-Whitney U test, KruskallWallis test, Spearman correlation) were used, as neither tau protein nor tau-pT181 was normally distributed.

\section{RESULTS}

CSF concentrations of tau protein, tau-pT181, and the taupT181/tau protein ratio for both SCJD and vCJD and the relevant control groups are shown in fig 1 . The diagnostic value of each of these markers is shown in table 1 .

Efficiency is defined as the number of true positive and true negative values expressed as a percentage of the total number of patients investigated. Cut off values for tau-pT181 and the tau-pT181/tau protein ratio were calculated using receiver operator curves; the cut off value used for tau protein was that previously described. ${ }^{4}$

The mean concentration of CSF tau-pT181 was significantly raised in VCJD patients compared with vCJD control patients ( $114 v 33 \mathrm{pg} / \mathrm{ml}, \mathrm{p}<0.001)$ and SCJD patients ( $114 v$ $61 \mathrm{pg} / \mathrm{ml}, \mathrm{p}<0.001)$. The mean concentration of tau-pT181 was raised in SCJD patients compared with SCJD controls (61 $v 35 \mathrm{pg} / \mathrm{ml}, \mathrm{p}=0.002$ ). There was no evidence of an effect of age, sex, or disease duration on the tau-pTl81 protein concentration in either SCJD or vCJD.

The mean concentration of CSF tau protein was significantly raised in VCJD and SCJD when compared with the appropriate control groups (952 v $106 \mathrm{pg} / \mathrm{ml}, \mathrm{p}<0.00 \mathrm{l}$; and $5120 \vee 367 \mathrm{pg} / \mathrm{ml}, \mathrm{p}<0.001$, respectively). CSF tau protein had the greatest efficiency for discriminating between both vCJD and SCJD from their respective control patients (table 1). There was a strong correlation between tau protein and tau-pT181 protein in vCJD patients and vCJD controls (Spearman's test, $\mathrm{p}<0.001$ ), no evidence of a correlation in sCJD patients $(\mathrm{p}=0.3)$, and slight evidence of a correlation in sCJD controls $(p=0.049)$.

The tau-pT181/tau protein ratios for vCJD and SCJD were significantly lower than in the appropriate control groups ( $119 \vee 279, \mathrm{p}<0.001 ; 12 \vee 128, \mathrm{p}<0.001$, respectively), but this ratio did not improve the diagnostic efficiency of CSF tau protein alone (table 1).

\section{DISCUSSION}

Patients with VCJD and SCJD had significantly raised concentrations of tau protein and tau-pT181 compared with control patients. Interestingly the concentration of CSF taupT181 was higher in vCJD patients than in SCJD or either group of control patients. As there was no correlation between tau-pT181 concentration and age or disease duration in either vCJD or SCJD, the difference in mean concentration between these two forms of CJD is unlikely to be explained by the younger age or the longer clinical course in vCJD patients.

The fact that the mean concentration of tau-pTl81 is higher in VCJD than in SCJD, in contrast to tau protein levels where the reverse is true, suggests that the factors governing the release or metabolism of tau protein and tau-pTl81 are different in these two forms of CJD. It is thought that tau protein is released into the CSF as a result of neuronal damage or death and that the magnitude of the concentrations reflects the extent and rate of this process. As SCJD has 
Table 1 Sensitivity, specificity, and efficiency values for CSF tau protein, tau-pT181 and tau-pT181/tau protein ratio in sporadic and variant CJD

\begin{tabular}{|c|c|c|c|}
\hline SCJD: & $\begin{array}{l}\text { Tau protein } \\
\geqslant 1300 \mathrm{pg} / \mathrm{ml}\end{array}$ & $\begin{array}{l}\text { Tau-pT181 } \\
\geqslant 50 \mathrm{pg} / \mathrm{ml}\end{array}$ & $\begin{array}{l}\text { Tau-pT181/tau protein ratio } \\
\leqslant 60\end{array}$ \\
\hline $\begin{array}{l}\text { Sensitivity (\%) } \\
\text { Specificity (\%) } \\
\text { Efficiency (\%) }\end{array}$ & $\begin{array}{l}84 \\
91 \\
88\end{array}$ & $\begin{array}{l}60 \\
61 \\
60\end{array}$ & $\begin{array}{l}88 \\
74 \\
81\end{array}$ \\
\hline VCJD: & $\begin{array}{l}\text { Tau protein } \\
\geqslant 400 \mathrm{pg} / \mathrm{ml}\end{array}$ & $\begin{array}{l}\text { Tau-pT181 } \\
\geqslant 70 \mathrm{pg} / \mathrm{ml}\end{array}$ & $\begin{array}{l}\text { Tau-pT181/tau protein ratio } \\
\leqslant 200\end{array}$ \\
\hline $\begin{array}{l}\text { Sensitivity (\%) } \\
\text { Specificity (\%) } \\
\text { Efficiency (\%) }\end{array}$ & $\begin{array}{l}90 \\
89 \\
90\end{array}$ & $\begin{array}{l}84 \\
86 \\
85\end{array}$ & $\begin{array}{l}86 \\
78 \\
83\end{array}$ \\
\hline
\end{tabular}

a more rapid clinical course than vCJD this would explain the higher mean concentrations of CSF tau protein in SCJD than in vCJD. The higher mean concentrations of CSF tau-pT181 in vCJD than in SCJD or in the control patients suggests that there are factors as yet unexplained that influence the phosphorylation of tau protein in vCJD patients. CSF taupT181 concentrations are increased in Alzheimer's disease, a condition associated with neurofibrillary tangles (NFTs), but there is no neuropathological evidence that vCJD patients have NFTs or any other tau inclusion bodies. ${ }^{11}$ At present, it is unclear whether this increase in tau-pT181 results from increased phosphorylation of tau protein or from a reduction or resistance to de-phosphorylation.

The mean tau-pTl81/tau protein ratio in vCJD was significantly lower than in the relevant control group but was not as efficient as the CSF tau protein alone in discriminating between these two groups of patients. Interestingly the tau-pT181/tau protein ratio is higher in vCJD than in SCJD and reflects the differences in magnitude of the two proteins in the two forms of CJD.

\section{Conclusions}

CSF tau-pT181 is the only surrogate marker investigated so far that is significantly raised in vCJD when compared with sCJD and controls. Despite this, measurement of CSF taupT181 or calculation of the tau-pT181/tau protein ratio does not improve the diagnostic accuracy of tau protein alone. Whether raised CSF tau-pT181 levels may be of diagnostic value in distinguishing between VCJD and SCJD in a clinical setting will require further investigation. Interestingly the observation that vCJD patients have higher concentrations of CSF tau-pT181 than patients with SCJD suggests that the pathogenic factors governing the phosphorylation of tau protein in these two conditions are different.

\section{ACKNOWLEDGEMENTS}

This study was funded by a grant from the Department of Health, UK (grant No 007/0075). The National CJD Surveillance Unit is funded by the Department of Health and the Scottish Home Office Department of Health.

\section{Authors' affiliations}

C A Goodall, M W Head, D Everington, J W Ironside, R S G Knight,

A J E Green, The National Creutzfeldt-Jakob Disease Surveillance Unit, Western General Hospital, Crewe Road, Edinburgh, UK

Competing interests: none declared

Correspondence to: Dr Alison Green, The National CJD Surveillance Unit, Western General Hospital, Crewe Road, Edinburgh EH4 2XU, UK; Alison.Green@ed.ac.uk

Received 16 February 2005

In revised form 4 April 2005

Accepted 10 April 2005

\section{REFERENCES}

1 Goedart M, Crowther RA, Garner CC. Molecular characterisation of microtubule-associated proteins tau and MAP2. Trends Neurosci 1991;14:193-9.

2 Buee L, Delacourte A. Comparative biochemistry of tau in progressive supranuclear palsy, corticobasal degeneration, FTDP-17 and Pick's disease. Brain Pathol 1999;9:681-93

3 Kapaki E, Kilidireas K, Paraskevas GP, et al. Highly increased CSF tau protein and decreased beta-amyloid (1-42) in sporadic CJD: a discrimination from Alzheimer's disease? J Neurol Neurosurg Psychiatry 2001;71:401-3.

4 Otto $M$, Wilffang J, Tumani $\mathrm{H}$, et al. Elevated levels of tau-protein in cerebrospinal fluid of patients with Creutzfeldt-Jakob disease. Neurosci Lett 1997;225:210-12.

5 Riemenschneider M, Wagenpfeil S, Vanderstichele H, et al. Phospho-tau/total tau ratio in cerebrospinal fluid discriminates Creutzfeldt-Jakob disease from other dementias. Mol Psychiatry 2003;8:343-7.

6 Van Everbroeck B, Green AJ, Vanmechelen E, et al. Phosphorylated tau in cerebrospinal fluid as a marker for Creutzfeldt-Jakob disease. J Neurol Neurosurg Psychiatry 2002;73:79-81.

7 Green AJE, Thompson EJ, Stewart GE, et al. Use of 14-3-3 and other brainspecific proteins in the CSF in the diagnosis of variant Creutzfeldt-Jakob disease. J Neurol Neurosurg Psychiatry 2001;70:744-8.

8 Will RG, Zeidler M, Stewart GE, et al. Diagnosis of new variant CreutzfeldtJakob disease. Ann Neurol 2000;47:575-82.

9 Ironside JW, Head MW, McCardle L, et al. Neuropathology of variant Creutzfeldt-Jakob disease. Acta Neurobiol Exp (Wars) 2002;62:175-82.

10 Will RG, Alperovitch A, POSER S, et al. Descriptive epidemiology of Creutzfeldt-Jakob disease in six European countries, 1993-1995. EU Collaborative Study Group for CJD. Ann Neurol 1998;43:763-67.

11 Ironside JW, Head MW, Bell JE, et al. Laboratory diagnosis of variant Creutzfeldt-Jakob disease. Histopathology 2000;37:1-9. 